\title{
Dynamic Crack Propagation in a Layered Medium Under Antiplane Shear
}

Chien-Ching Ma

Professor.

\author{
Yi-Shyong Ing
}

Graduate Student

Department of Mechanical Engineering, National Taiwan University

Taipei, Taiwan 10617

Republic of China
In this study, the transient analysis of dynamic antiplane crack propagation with a constant velocity in a layered medium is investigated. The individual layers are isotropic and homogeneous. Infinite numbers of reflected cylindrical waves, which are generated from the interface of the layered medium, will interact with the propagating crack and make the problem extremely difficult to analyze. A useful fundamental solution is proposed in this study, and the solution can be determined by superposition of the fundamental solution in the Laplace transform domain. The proposed fundamental problem is the problem of applying exponentially distributed traction (in the Laplace transform domain) on the propagating crack faces. The Cagniard's method for Laplace inversion is used to obtain the transient solution in time domain. The exact closed-form transient solutions of dynamic stress intensity factors are expressed in compact formulations. These solutions are valid for an infinite length of time and have accounted for contributions from all the incident and reflected waves interaction with the moving crack tip. Numerical results of dynamic stress intensity factors for the propagation crack in layered medium are evaluated and discussed in detail.

\section{Introduction}

Most of the analyses done regarding cracked bodies are quasistatic. There are numerous situations that the material inertia becomes significant and must be taken into account in the analyses. The question of whether or not inertial effects are significant depends on the loading conditions and the geometrical configuration of the body. Inertial effects can arise either from applying dynamic loading on a cracked solid or from rapid crack propagation. The inherent time dependence of a dynamic fracture process results in mathematical models that are more complex than equivalent quasi-static models. However, there is substantial interest in the dynamic fracture problem due to its importance in many engineering applications. The relevant applications are NDE of layered media, rupture due to earthquakes in earth's crust, plate tectonics, composite materials, and functionally graded materials.

The main purpose in solving problems concerned with dynamic crack propagation is to determine the dependence of the crack-tip field characterizing parameters on the applied loading and on the configuration of the body. The investigation of a propagation crack in a brittle solid began with the pioneering analysis of Yoffe (1951). She considered a steady-state crack growth problem of a crack of fixed length propagating in an infinite elastic body subjected to a uniform remote tensile loading normal to the crack line. Although this is a physically unrealistic problem, it did at least provide an indication of the influence of crack speed on the stress state of a rapidly propagating crack. Craggs (1960) considered a semi-infinite crack extending at constant speed, with the crack face loading moving with the same speed as the crack tip in such a way that the entire deformation field is constant as seen by an observer moving with the

Contributed by the Applied Mechanics Division of THE AMERICAN SocIETY of MECHANICAL ENGINEERS for publication in the ASME JOURNAL OF APPLIED MECHANICS.

Discussion on this paper should be addressed to the Technical Editor, Professor Lewis T. Wheeler, Department of Mechanical Engineering, University of Houston, Houston, TX 77204-4792, and will be accepted until four months after final publication of the paper itself in the ASME JourNaL of APPLIED MECHANICS.

Manuscript received by the ASME Applied Mechanics Division, July 10, 1995; final revision, Aug. 26, 1996. Associate Technical Editor: J. W. Ju. crack tip. The self-similar dynamic crack propagation problem was contributed by Broberg (1960) who was among the first to present detailed analyses of crack propagation as a transient process. He solved the dynamic problem of a crack that suddenly grows from zero length at a constant speed. Baker (1962) subsequently generalized Broberg's solution to include a finite initial crack. Although the above mentioned artificial solutions have no direct application, they also have provided useful insights and continue to be used today for the assessment of dynamic numerical analyses.

In a series of papers, Freund (1972a, 1972b, 1973, 1974) developed important analytical methods for evaluation of the transient stress field of a propagating crack in a two-dimensional geometric configuration under quite general dynamic loading situation. These particular cases analyzed by Freund are also self-similar, but they are solved by means of integral transform methods rather than by direct application to similarity arguments. An indirect analytical approach proposed by Freund based on superposition over a fundamental solution, which opens a way for analysis of certain problems of crack propagation at nonuniform speed. Based on the superposition method proposed by Freund, a series of problems for nonplanar crack propagation in an infinite domain was solved by $\mathrm{Ma}$ and Burgers (1986, 1987, 1988) and $\mathrm{Ma}(1988,1990)$. The structure of the near-tip field of crack propagation at nonuniform speeds was discussed in detail by Freund and Rosakis (1992). A representation of the crack-tip field was obtained in the form of an expansion about the crack tip in powers of radial coordinate, with the coefficients depending on the time rates of change of crack-tip speed and stress intensity factor. This representation was used to interpret some experimental observations and some estimates were made of the practical limits of using a stress intensity factor field alone to characterize the local fields. A thorough summary of the application of the main direct methods of analysis for transient problem in dynamic fracture has been given by Freund (1990).

Most of the solved dynamic fracture problems are regarded as a crack subjected to applying a uniformly distributed dynamic loading on the crack faces or subjected to incident plane waves. For the above-mentioned problems, either the direct application 
of the well-known Wiener-Hopf technique is used or the superposition method proposed by Freund is performed to solve the problems. However, if a crack is subjected to incident nonplanar waves, none of the known methods can be used to obtain the transient solutions. Recently Tsai and Ma (1992) proposed a new fundamental solution to overcome these difficulties. The fundamental problem they considered is an exponentially distributed traction applied on crack faces and the solution is constructed by superposition of the fundamental solution in the Laplace transform domain. Tsai and $\mathrm{Ma}$ (1992) used this new fundamental solution to obtain the transient solution for a stationary semi-infinite crack subjected to a suddenly applied dynamic inplane body force in an unbounded medium. This alternative fundamental solution is also successfully applied towards solving more complicated transient problems (Tsai and $\mathrm{Ma}$, 1993a, 1993b; Ma and Chen, 1994) for a subsurface stationary inclined crack subjected to dynamic loadings.

Determination of the dependence of the dynamic stress intensity factor on body configuration and applied loading during rapid crack propagation is a principal objective in dynamic fracture. Analytical results for configurations with boundaries other than the crack faces are rare. It was noted that after the stress waves reflected from the boundaries arrived at the crack tip, the nature of crack propagation changed. The interaction of stress waves with propagating cracks provides a way of altering the crack-tip stress field, and thus provide the basis for the crack branching phenomenon. Dynamic analysis of a mode III crack in a strip was discussed in a paper by Nilsson (1973) and Ma and Ing (1995). They considered the problem of a semi-infinite crack in a strip of finite width subjected to a uniformly distributed static load and propagating with a constant velocity, only the stress intensity factor and its time-dependence was determined.

The mechanical behavior of many newly developed multiphase materials are mainly controlled by the response of the interfaces. Many researchers have devoted to investigating the field of dynamic debonding along a bimaterial interface recently. Yang et al. (1991) examined singular fields around a running crack along the interface between two anisotropic substrates. The stress singularities and the angular stress distributions near a propagating interface crack in different transonic regimes for both antiplane and in-plane cases are determined by Yu and Yang (1994, 1995). Yang and Shih (1994) characterized the transition of the elastic moduli across the bimaterial interface by introducing a nonhomogeneous interlayer. The interlayer model provides some important concepts of interface fracture mechanics.

Since the interaction of stress waves with a propagating crack is an important event in dynamic fracture research, we shall investigate it in greater detail in the following. In this study, we will extend the methodology that was used successfully in solving dynamic stationary crack problem to construct the transient solution for crack propagation in the configuration of a layered medium. The problem considered in this study is a horizontal semi-infinite crack propagating with constant velocity in an infinitely long strip with finite width for a layered medium as shown in Fig. 1. In analyzing the above-mentioned

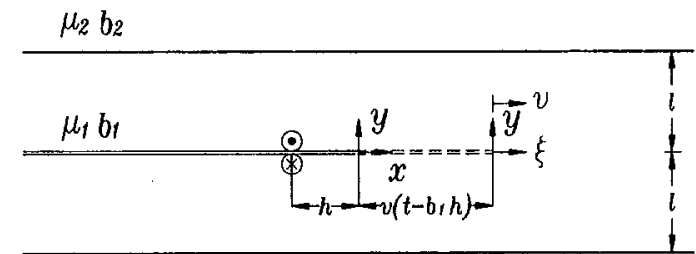

$\mu_{2} b_{2}$

Fig. 1 Configuration and coordinate systems of a propagating crack in a layered medium problems, the interface boundaries and propagating crack are considered into the analysis. The interaction of infinitely many reflected waves generated from the interface boundaries and the propagating crack must be taken into account which will make the analysis extremely difficult. A useful fundamental solution is proposed to overcome these difficulties. This proposed fundamental solution is successfully applied towards solving the problem and is demonstrated as an efficient methodology to solve similar problems. Since the stress intensity factor is the key parameter in characterizing dynamic crack grows, we will focus our attention mainly on the determination of the dynamic stress intensity factor.

\section{Required Fundamental Solutions}

As usual in problems of the type considered here, superposition of solutions plays a significant role. The solutions of the problem considered in this study can be determined by superposition of the following problems. Problem A treats a dynamic concentrated loading acting on semi-infinite crack faces in an unbounded medium at time $t=0$, at $t=t_{f}$, a new crack propagates out of the original semi-infinite crack with constant velocity, which induces incident cylindrical waves propagating toward the plane that will eventually define the interface boundary. In problem $\mathbf{B}$, an interface in an unbounded medium is considered in which the interface is subjected to incident waves which are equal to the corresponding waves generated from the crack tip in problem A. Problem $\mathrm{C}$ considers an infinite body containing a propagating semi-infinite crack in which the crack is subjected to the reflected waves which are generated by the interface boundary in problem $\mathrm{B}$. The three fundamental problems A, B, and C which are superimposed to obtain the solution for propagating crack in a layered medium interaction with stress waves. The reflected waves induced from the interface boundary as indicated in problem $\mathrm{B}$, can be obtained by constructing the solution for incident cylindrical waves interaction with a interface in a layered medium. While problem $\mathrm{A}$ is a well-known problem for a semi-infinite crack propagating in an unbounded medium and many efforts have been devoted to analyze this problem.

Problem $C$ in the above mentioned three fundamental problems is the only one which needs careful analysis. The problem we will deal with is the interaction of the propagating crack with cylindrical reflected waves, which causes the only difficulty in this investigation. For most of the dynamic problems, the propagating waves can be represented in an exponential functional form in the Laplace transform domain of time. The reflected and diffracted waves generated by the half-plane boundary and by the crack can be constructed by the proposed superposition method if the responses toward an applied exponentially distributed traction on boundaries in the Laplace transform domain can be obtained preliminarily. The superposition scheme proposed in this study, unlike usual superposition methods which are performed in the time domain, is performed in the Laplace transform domain.

Consider the fundamental problem for a propagating semiinfinite crack with constant velocity in an unbounded medium. The solution for an exponentially distributed loading applied at the crack faces in the Laplace transform domain will be referred to as the fundamental solution. The problem can be viewed as a half-plane problem with the material occupying the region $y$ $\geq 0$, and subjected to the following mixed boundary conditions in the Laplace transform domain:

$$
\begin{gathered}
\bar{\gamma}_{y z}(\xi, 0, s)=e^{s \eta \xi} \quad-\infty<\xi<0, \\
\bar{w}(\xi, 0, s)=0 \quad 0<\xi<\infty
\end{gathered}
$$

where $s$ is the Laplace transform parameter and $\eta$ is a constant. The coordinate $\xi$ is fixed with respect to the moving crack tip, that is $\xi=x-v t$. The overbar symbol is used for denoting the 
transform on time $t$. The one-sided Laplace transform with respect to time and the two-sided Laplace transform with respect to $\xi$ are defined by

$$
\begin{gathered}
\bar{w}(\xi, y, s)=\int_{0}^{\infty} w(\xi, y, t) e^{-s t} d t \\
\tilde{w}(\lambda, y, s)=\int_{-\infty}^{\infty} \bar{w}(\xi, y, s) e^{-s \lambda \xi} d \xi .
\end{gathered}
$$

This fundamental problem can be solved by using the standard transform method and the Wiener-Hopf technique. The governing equation can be represented by the two-dimensional wave equation

$$
\frac{\partial^{2} w}{\partial x^{2}}+\frac{\partial^{2} w}{\partial y^{2}}=b^{2} \frac{\partial^{2} w}{\partial t^{2}}
$$

where $b$ is the slowness of the shear wave and is given by

$$
b=1 / v_{s}=\sqrt{\rho / \mu},
$$

in which $w(x, y, t)$ is the displacement normal to the $x y$-plane; $v_{s}$ is the shear wave speed, $\mu$ and $\rho$ are the respective shear modulus and the mass density of the material. The nonvanishing shear stresses are

$$
\tau_{y z}=\mu \frac{\partial w}{\partial y}, \quad \tau_{x z}=\mu \frac{\partial w}{\partial x} .
$$

In analyzing this problem, it is convenient to express the governing equation in the moving coordinates $\xi-y$ as follows:

$$
\left(1-b^{2} v^{2}\right) \frac{\partial^{2} w}{\partial \xi^{2}}+\frac{\partial^{2} w}{\partial y^{2}}+2\left(b^{2} v\right) \frac{\partial^{2} w}{\partial \xi \partial t}-b^{2} \frac{\partial^{2} w}{\partial t^{2}}=0 .
$$

This fundamental problem can be solved by the application of integral transforms. Applying the one-sided Laplace transform over time, the two-sided Laplace transform over $\xi$ under the restriction of $\operatorname{Re}(\eta)>\operatorname{Re}(\lambda)$, finally the Wiener-Hopf technique is implemented. The solutions of stresses and displacement in the transform domain for the boundary conditions (2.1) and (2.2) are

$$
\begin{gathered}
\bar{\tau}_{y z}(\xi, y, s)=\frac{1}{2 \pi i} \int_{\Gamma_{\lambda}} \frac{\alpha_{+}^{*}(\lambda) e^{-s\left(\alpha^{*} y-\lambda \xi\right)}}{\alpha_{*}^{*}(\eta)(\eta-\lambda)} d \lambda, \\
\bar{\tau}_{x z}(\xi, y, s)=-\frac{1}{2 \pi i} \int_{\Gamma_{\lambda}} \frac{\lambda e^{-s\left(\alpha^{*} y-\lambda \xi\right)}}{\alpha_{+}^{*}(\eta)(\eta-\lambda) \alpha_{-}^{*}(\lambda)} d \lambda, \\
\bar{W}(\xi, y, s)=-\frac{1}{2 \pi i} \int_{\Gamma_{\lambda}} \frac{e^{-s\left(\alpha^{*} y-\lambda \xi\right)}}{\mu s \alpha_{+}^{*}(\eta)(\eta-\lambda) \alpha_{-}^{*}(\lambda)} d \lambda,
\end{gathered}
$$

where

$$
\alpha^{*}(\lambda)=\sqrt{b+\lambda(1-b v)} \sqrt{b-\lambda(1+b v)}=\alpha_{+}^{*}(\lambda) \alpha^{*}(\lambda) .
$$

The corresponding result of the dynamic stress intensity factor in the Laplace transform domain is

$$
\begin{aligned}
\vec{K}(s) & =\lim _{\xi \rightarrow 0} \sqrt{2 \pi \xi} \bar{\tau}_{y z}(\xi, 0, s) \\
& =-\frac{\sqrt{2} \sqrt{1-b v}}{\sqrt{s} \alpha *(\eta)}
\end{aligned}
$$

\section{Transient Analysis for Propagating Crack in a Layered Media}

Consider the problem of a semi-infinite crack in an infinitely long strip with finite width $2 l$ of a layered medium as shown in Fig. 1. At time $t=0$, a pair of equal and opposite concentrated antiplane dynamic loadings with magnitude $p$ are applied at the crack faces with a distance $h$ from the crack tip, the crack starts to propagate with constant velocity $v$ at a delay time that the wave reach the crack tip (i.e., $t_{f}=b_{1} h$ ) in the $x$-direction along the symmetry line $y=0$ of the strip. The time dependence of the loading is represented by the Heaviside step function $H(t)$. In this problem, the cylindrical wave induced from the dynamic concentrated loading and the diffracted waves generated from the propagating crack tip will be reflected from horizontal interface boundaries, these waves will interact with the propagating crack at some later time. The major difficulty in analyzing this problem will be the one that deals with the interaction of reflected waves with the propagating crack and the superposition technique of the fundamental solutions in the Laplace transform domain will be used in the analysis. The transient solutions are composed of incident field, reflected field and diffracted field, which will be denoted by superscripts of $i, r$, and $d$, respectively. Before the time that the $i$ and $d$ waves reflected from the interface boundaries of the strip, the problem can be considered as a semi-infinite crack propagating in an unbounded medium. In the following analysis, the solution is valid for the case that crack starts to propagating before the waves (i.e., $i$ wave and $d$ wave) generated from the stationary crack returns to the stationary crack (i.e., $t_{f}<2 b l$ ) and $b_{1}<b_{2}$.

The incident field of the cylindrical wave generated by the concentrated loading expressed in the Laplace transform domain can be obtained as follows:

$$
\bar{\tau}_{y z}(x, 0, s)=\frac{1}{2 \pi i} \int_{\Gamma_{\mathrm{\lambda}}}-p e^{s \lambda(x+h)} d \lambda .
$$

The applied traction on the crack face as indicated in (3.1), has a functional form $e^{s \lambda x}$. Since the solutions of applying traction $e^{s \eta x}$ on crack faces in the Laplace transform domain have been solved in Section 2 by setting $v=0$, the diffracted field generated from the stationary semi-infinite crack can be constructed by superimposing the incident wave traction that is equal to (3.1). When we combine (2.5) (by setting $v=0$ ) and (3.1), the solution of diffracted wave in the Laplace transform domain can be expressed as follows:

$$
\begin{aligned}
\bar{\tau}_{y z}^{s}(x, y, s)=\frac{1}{2 \pi i} \int_{\Gamma_{\lambda}}-p\{ & \frac{1}{2 \pi i} \int_{\Gamma_{\eta_{2}}} \frac{\left(b_{1}+\eta_{2}\right)^{1 / 2}}{\left(\lambda-\eta_{2}\right)\left(b_{1}+\lambda\right)^{1 / 2}} \\
& \left.\times e^{s \lambda h} e^{-s\left(\alpha_{1} y-\eta_{2} x\right)} d \eta_{2}\right\} d \lambda .
\end{aligned}
$$

By using the Cagniard-de Hoop method of Laplace inversion, the incident and diffracted stress fields in time domain for the stationary crack are obtained as follows:

$$
\tau_{y z}^{i}(x, y, t)=\frac{-p t \sin \theta}{\pi r\left(t^{2}-b_{1}^{2} r^{2}\right)^{1 / 2}} H\left(t-b_{1} r\right),
$$

$$
\begin{aligned}
\tau_{y z}^{d}(x, y, t)=\frac{p}{2 \pi^{2}} \int_{b_{1} h}^{t-b_{1} r_{2}} & \operatorname{Re}\left[H\left(\eta_{1}^{+}, \eta_{2}^{+}\right) \frac{\partial \eta_{1}^{+}}{\partial t_{1}} \frac{\partial \eta_{2}^{+}}{\partial t_{2}}\right. \\
& \left.-H\left(\eta_{1}^{-}, \eta_{2}^{+}\right) \frac{\partial \eta_{1}^{-}}{\partial t_{1}} \frac{\partial \eta_{2}^{+}}{\partial t_{2}}\right] d t_{1}
\end{aligned}
$$

where

$$
\begin{gathered}
\eta_{\mathrm{t}}^{ \pm}=-\frac{t_{1}}{h} \pm i \varepsilon, \\
\eta_{2}^{ \pm}=-\frac{t_{2}}{r_{2}} \cos \theta_{2} \pm i\left(\frac{t_{2}^{2}}{r_{2}^{2}}-b_{1}^{2}\right)^{1 / 2} \sin \theta_{2}, \\
r=\left[(x+h)^{2}+y^{2}\right]^{1 / 2}, \quad \theta=\cos ^{-1}[(x+h) / r], \\
r_{2}=\left(x^{2}+y^{2}\right)^{1 / 2}, \quad \theta_{2}=\cos ^{-1}\left(x / r_{2}\right),
\end{gathered}
$$




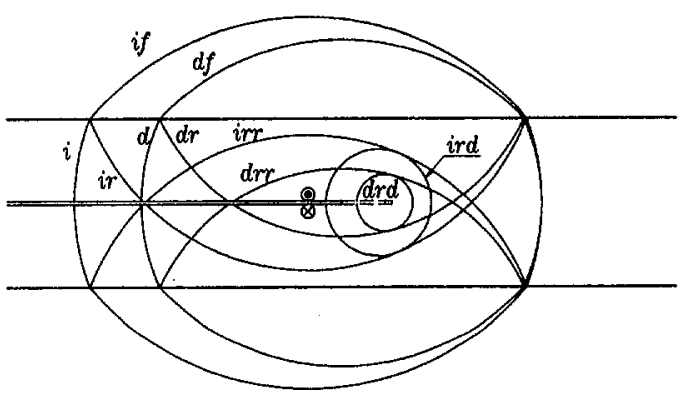

Fig. 2 Configuration of wave fronts in the layered medium after the crack propagates with constant velocity

$$
\begin{gathered}
H\left(\eta_{1}, \eta_{2}\right)=\frac{\left(b_{1}+\eta_{2}\right)^{1 / 2}}{\left(\eta_{1}-\eta_{2}\right)\left(b_{1}+\eta_{1}\right)^{1 / 2}}, \\
t=t_{1}+t_{2} .
\end{gathered}
$$

The corresponding dynamic stress intensity factor induced by diffracted wave will be

$$
K^{s}(t)=p \sqrt{\frac{2}{\pi h}} H\left(t-b_{1} h\right) .
$$

The dynamic stress intensity factor shown in (3.5) jumps from zero at the instant that the cylindrical wave generated by the concentrated loading reaches the crack tip. At time $t=t_{f}=$ $b_{1} h$, the dynamic stress intensity factor reaches its critical value and the crack starts to propagate with constant velocity $v$ along the line $y=0$. The transient full-field analysis for a propagating crack just mentioned above has also been solved by $\mathrm{Ma}$ and Chen (1992) by using the method proposed by Freund (1972b). In their investigations, the transient solution for constant-speed crack propagation is obtained by determining a fundamental solution for a concentrated force appearing through the moving crack tip, and then building up the general solution by superposition. In this study, a more direct and simple methodology will be used to solve this problem. We consider the transient problem of a semi-infinite crack propagates at $t=t_{f}=b_{1} h$ with symmetric concentrated loading applied only on the original crack faces $-\infty<x<0$. The applied symmetric concentrated loading $p$

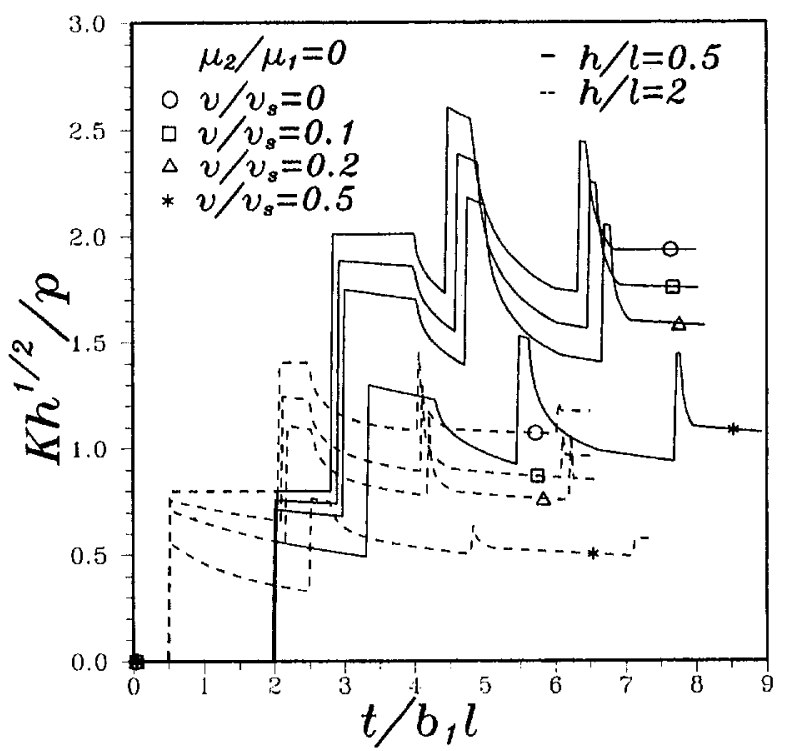

Fig. 3 Transient response of the dynamic stress intensity factor for various crack propagating speed

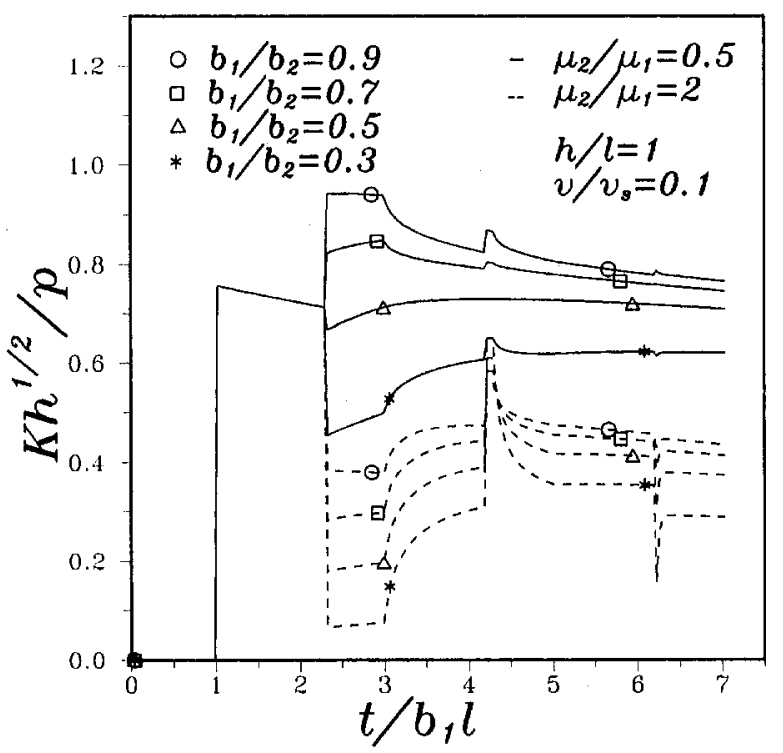

Fig. 4 Transient response of the dynamic stress intensity factor for different ratio of shear wave speed

on the original crack faces written in the Laplace transform domain for the moving coordinate system will have the following form:

$$
\bar{\tau}_{y z}(\xi, 0, s)=\frac{1}{2 \pi i} \int_{\Gamma_{\lambda}} \frac{p d}{(\lambda-d)} e^{s h\left(1-b_{1} v\right) \lambda+s \lambda \xi} d \lambda,
$$

in which $d=1 / v$ is the slowness of the crack velocity and $\xi$ $=x-v t$. The applied traction on crack faces as expressed in (3.6), has the functional form $e^{s \lambda \xi}$. Since the Laplace transform solutions of applying traction $e^{s \eta \xi}$ on crack faces have been solved in the previous section, the diffracted field generated from the propagating crack tip can be constructed by superimposing the fundamental solution and the stress distribution in (3.6). The result of shear stress expressed in the Laplace transform domain will be

$$
\begin{aligned}
& \bar{\tau}_{y z}^{d}(\xi, y, s)=\frac{1}{2 \pi i} \int_{\Gamma_{\eta_{1}}} \frac{p d}{\left(\eta_{1}-d\right)} e^{s h\left(1-b_{1} v\right) \eta_{1}} \\
& \quad \times\left\{\frac{1}{2 \pi i} \int_{\Gamma_{\eta_{2}}} \frac{\alpha_{1+}^{*}\left(\eta_{2}\right)}{\left(\eta_{1}-\eta_{2}\right) \alpha_{1+}^{*}\left(\eta_{1}\right)} e^{-s\left[\alpha_{1}^{*} y-\eta_{2} \xi \mid\right.} d \eta_{2}\right\} d \eta_{1},
\end{aligned}
$$

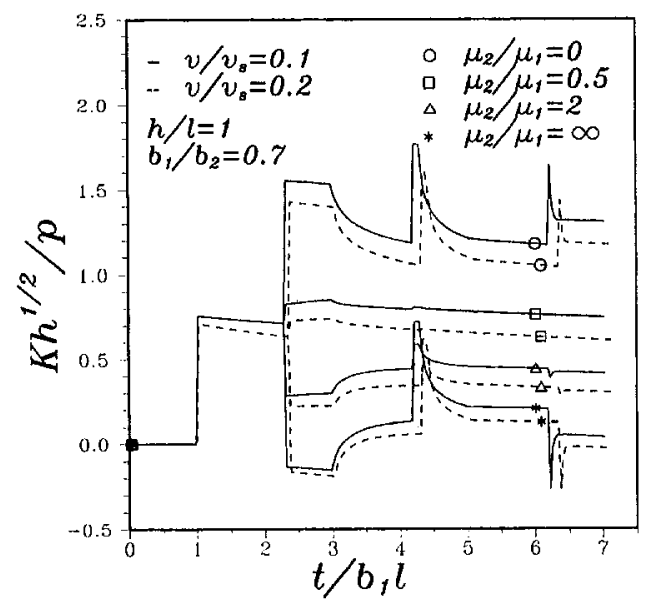

Fig. 5 Transient response of the dynamic stress intensity factor for different ratio of shear modulus of the layered medium 
The exact transient solutions for a propagating crack in an unbounded medium in time domain can be obtained by inverting the Laplace transform domain of (3.7). The result is

$$
\begin{aligned}
\tau_{y z}^{d}(\xi, y, t)=\frac{-1}{2 \pi^{2}} \int_{b_{1} h}^{t-t_{d}} & \operatorname{Re}\left[G\left(\eta_{1}^{+}, \eta_{2}^{+}\right) \frac{\partial \eta_{1}^{+}}{\partial t_{1}} \frac{\partial \eta_{2}^{+}}{\partial t_{2}}\right. \\
& \left.-G\left(\eta_{1}^{-}, \eta_{2}^{+}\right) \frac{\partial \eta_{1}^{-}}{\partial t_{1}} \frac{\partial \eta_{2}^{+}}{\partial t_{2}}\right] d t_{1},
\end{aligned}
$$

where

$$
\begin{gathered}
G\left(\eta_{1}, \eta_{2}\right)=\frac{p d \alpha_{1+}^{*}\left(\eta_{2}\right)}{\left(\eta_{1}-d\right) \alpha_{1+}^{*}\left(\eta_{1}\right)\left(\eta_{1}-\eta_{2}\right)} \\
\eta_{1}^{ \pm}=\frac{-t_{1}}{h\left(1-b_{1} v\right)} \pm i \varepsilon \\
\eta_{2}^{ \pm}=\frac{-\left(\xi t_{2}+b_{1}^{2} v y^{2}\right) \pm i y \sqrt{\left.t_{2}^{2}-b_{1}^{2}\left[y^{2}+\left(\xi+v t_{2}\right)^{2}\right)\right]}}{\xi^{2}+\left(1-b_{1}^{2} v^{2}\right) y^{2}} \\
\frac{\partial \eta_{2}^{+}}{\partial t_{2}}=\frac{-\xi+i y\left\{t_{2}^{2}-b_{1}^{2}\left[y^{2}+\left(\xi+v t_{2}\right)^{2}\right]\right\}^{-1 / 2}\left[t_{2}\left(1-b_{1}^{2} v^{2}\right)-b_{1}^{2} v \xi\right]}{\xi^{2}+\left(1-b_{1}^{2} v^{2}\right) y^{2}} \\
t_{d}=\frac{b_{1}\left\{b_{1} v \xi+\left[\xi^{2}+\left(1-b_{1}^{2} v^{2}\right) y^{2}\right]^{1 / 2}\right\}}{1-b_{1}^{2} v^{2}} \\
t_{1}+t_{2}=t .
\end{gathered}
$$

The dynamic stress intensity factor for a propagating crack at an infinite medium can also be constructed by a similar manner. The result in the Laplace transform domain can be obtained from (2.8) and (3.6) and is expressed as follows:

$$
\begin{aligned}
K^{d}(s)=\frac{1}{2 \pi i} \int_{\Gamma_{\lambda}} \frac{p d}{(\lambda-d)} e^{s h\left(1-b_{1} v\right) \lambda} & \\
& \times\left\{\frac{-\sqrt{2} \sqrt{1-b_{1} v}}{\sqrt{s} \alpha_{1+}^{*}(\lambda)}\right\} d \lambda .
\end{aligned}
$$

The inversion Laplace transform of (3.9) will have the following form:

$$
\begin{aligned}
& K^{d}(t)=p \sqrt{\frac{2}{\pi\left[v\left(t-b_{1} h\right)+h\right]}} \\
& \times\left(1-b_{1} v\right)^{1 / 2} H\left(t-b_{1} h\right) .
\end{aligned}
$$

The expression for $K^{d}(t)$ in (3.10) has the interesting form of the product of a function of the crack velocity $\left(1-b_{1} v\right)^{1 / 2}$ and the corresponding stationary crack solution $K^{s}(t)$ in (3.5) with a distance $v\left(t-b_{1} h\right)+h$ from the crack tip. The value $\left(1-b_{1} v\right)^{1 / 2}$ is an universal function which depends only on crack speed and material properties. After some later time, the incident wave ( $i$ wave) generated from the dynamic concentrated loading and the diffracted wave ( $d$ wave) radiated out from the propagating crack will be reflected from the interface which will be indicated as the $i r$ and $d r$ wave, respectively. The solutions for reflected waves generated from the interface expressed in the Laplace transform domain can be obtained as follows:

$$
\begin{array}{r}
\bar{\tau}_{y z}^{i r+d r}(\xi, y, s)=\frac{1}{4 \pi^{2}} \iint \frac{\gamma_{1 / 2}^{*}\left(\eta_{2}\right) p d \alpha_{1+}^{*}\left(\eta_{2}\right)}{\left(\eta_{1}-d\right)\left(\eta_{1}-\eta_{2}\right) \alpha_{1+}^{*}\left(\eta_{1}\right)} \\
\times e^{s h\left(1-b_{1} v\right) \eta_{1} e^{s \alpha_{1}^{*}(y-2 l)+s \eta_{2} \xi} d \eta_{2} d \eta_{1}}
\end{array}
$$

where

$$
\gamma_{1 / 2}^{*}=\frac{\mu_{1} \alpha_{1}^{*}-\mu_{2} \alpha_{2}^{*}}{\mu_{1} \alpha_{1}^{*}+\mu_{2} \alpha_{2}^{*}} .
$$

The induced dynamic stress intensity factor by reflected $i r$ and $d r$ waves can be obtained by setting $y=0$ in (3.11) and the fundamental solution expressed in (2.8). The result for the stress intensity factor expressed in the Laplace transform domain will have the following form:

$$
\begin{array}{r}
\bar{K}^{i r d+d r d}(s)=\frac{1}{4 \pi^{2}} \iint \frac{p d \sqrt{2} \sqrt{1-b_{1} v} \gamma_{1 / 2}^{*}\left(\eta_{2}\right)}{\sqrt{s}\left(\eta_{1}-d\right)\left(\eta_{1}-\eta_{2}\right) \alpha_{1+}^{*}\left(\eta_{1}\right)} \\
\quad \times e^{s h\left(1-b_{1} v\right) \eta_{1}-2 s \alpha_{1}^{*}\left(\eta_{2}\right) l} d \eta_{2} d \eta_{1} .
\end{array}
$$

The dynamic stress intensity factor expressed in time domain will be

$$
\begin{aligned}
K^{i r d}(t)=\frac{-\sqrt{2} p d \sqrt{1-b_{1} v}}{\pi^{3 / 2}} \int_{t_{i r l}}^{t} \frac{1}{\sqrt{t-\tau}} \\
\quad \times \operatorname{Im}\left\{\frac{\gamma_{1 / 2}^{*}\left(\lambda^{+}\right) \frac{\partial \lambda^{+}}{\partial t}}{\left(\lambda^{+}-d\right) \alpha_{+}^{*}\left(\lambda^{+}\right)}\right\}_{t=\tau} d \tau,
\end{aligned}
$$

where

$$
\begin{aligned}
\lambda^{ \pm} & =\frac{-h\left(1-b_{1} v\right) t-4 b_{1}^{2} v l^{2} \pm i 2 l \sqrt{t^{2}-b_{1}^{2}\left\{\left[v t+h\left(1-b_{1} v\right)\right]^{2}+4 l^{2}\right\}}}{h^{2}\left(1-b_{1} v\right)^{2}+4 l^{2}\left(1-b_{1}^{2} v^{2}\right)} \\
t_{i r d} & =\frac{b_{1}^{2} v h\left(1-b_{1} v\right)+b_{1} \sqrt{h^{2}\left(1-b_{1} v\right)^{2}+4 l^{2}\left(1-b_{1}^{2} v^{2}\right)}}{1-b_{1}^{2} v^{2}}
\end{aligned}
$$

and

$$
\begin{aligned}
K^{d r d}(t) & =\frac{1}{2 \pi^{2}} \int_{b_{1} h+t_{d r d}^{\prime}}^{t} \int_{b_{1} h}^{\tau-t_{d r d}^{\prime}} \frac{\sqrt{2\left(1-b_{1} v\right)}}{\sqrt{\pi(t-\tau)}} \\
\times \operatorname{Re}\left[\frac{\gamma_{1 / 2}^{*}\left(\eta_{2}^{+}\right) G\left(\eta_{1}^{+}, \eta_{2}^{+}\right)}{\alpha_{1+}^{*}\left(\eta_{2}^{+}\right)} \frac{\partial \eta_{1}^{+}}{\partial t_{1}} \frac{\alpha \eta_{2}^{+}}{\partial t_{2}}\right. & \\
& \left.-\frac{\gamma_{1 / 2}^{*}\left(\eta_{2}^{+}\right) G\left(\eta_{1}^{-}, \eta_{2}^{+}\right)}{\alpha_{1+}^{*}\left(\eta_{2}^{+}\right)} \frac{\partial \eta_{1}^{-}}{\partial t_{1}} \frac{\alpha \eta_{2}^{+}}{\partial t_{2}}\right]_{t=\tau} d t_{1} d \tau
\end{aligned}
$$

where

$$
\begin{gathered}
\eta_{1}^{ \pm}=\frac{-t_{1}}{h\left(1-b_{1} v\right)} \pm i \varepsilon, \\
\eta_{2}^{+}=\frac{-2 b_{1}^{2} v l \pm i \sqrt{\left(1-b_{1}^{2} v^{2}\right) t_{2}^{2}-4 l^{2} b_{1}^{2}}}{2 l\left(1-b_{1}^{2} v^{2}\right)}, \\
t_{d r d}^{\prime}=\frac{-2 l b_{1}}{\sqrt{\left(1-b_{1}^{2} v^{2}\right)}} \\
t_{1}+t_{2}=t .
\end{gathered}
$$

Finally, the complete transient solutions of dynamic stress intensity factor that account for the contributions of all the reflected and diffracted waves are obtained explicitly. The solutions can be simplified into a very compact form as follows:

$$
\begin{aligned}
K(t)=K^{0}(t)+\sum_{m=1}^{\infty} 2 K^{m}(t) & +\sum_{n=1}^{\infty} 2^{n} K^{0, n}(t) \\
& +\sum_{m=1}^{\infty} \sum_{n=1}^{\infty} 2^{n+1} K^{m, n}(t),
\end{aligned}
$$

where

$$
\begin{aligned}
K^{0}(t)=p \sqrt{\frac{2}{\pi\left[v\left(t-b_{1} h\right)+h\right]}} \\
\times\left(1-b_{1} v\right)^{1 / 2} H\left(t-b_{1} h\right),
\end{aligned}
$$




$$
\begin{aligned}
K^{m}(t)= & \frac{p \sqrt{2\left(1-b_{1} v\right)}}{\pi^{3 / 2}} \int_{t_{v, m}}^{t} \frac{1}{\sqrt{t-\tau}} \\
& \times \operatorname{Im}\left\{\frac{\left[\gamma_{1 / 2}^{*}\left(\eta_{1, m}^{+}\right)\right]^{m}}{\left(1-\eta_{1, m}^{+} v\right) \alpha_{1+}^{*}\left(\eta_{1, m}^{+}\right)} \frac{\partial \eta_{1, m}^{+}}{\partial t_{1}}\right\}_{t_{1}=\tau} d \tau, \\
K^{m, n}(t)= & \frac{2 p \sqrt{2\left(1-b_{1} v\right) i^{a}}}{\sqrt{\pi(2 \pi i)^{n+1}}} \int_{t_{v, m}+n t_{t, r}}^{t} \int_{t_{u, m}}^{a_{1}} \int_{t_{r, r}}^{a_{2}} \int_{t_{r}}^{a_{3}} \ldots \\
& \int_{t_{r r}}^{a_{n}} \frac{1}{\sqrt{t-\tau}} \operatorname{SIFV}_{m, n} d t_{n} d t_{n-1} \ldots d t_{1} d \tau,
\end{aligned}
$$

in which

$$
\begin{gathered}
a_{1}=\tau-n t_{r r}, \\
a_{j}=\tau-t_{1}-t_{2}-\ldots-t_{j-1}-(n-j+1) t_{r r}, \\
\quad j=2,3, \ldots, n \\
q=0 \quad \text { when } n=1,3,5, \ldots ; \\
q=1 \quad \text { when } n=2,4,6, \ldots
\end{gathered}
$$

propagating speed. We can see that the stress intensity factor is large when the propagating speed is low. Figure 4 shows the dynamic stress intensity factor for different ratio of shear wave speed. It indicates that the stress intensity factor is small when the magnitude of the ratio $b_{1} / b_{2}$ is low. Figure 5 shows the transient dynamic stress intensity factor for different ratio of shear modulus of the layered medium, in which $\mu_{2} / \mu_{1}=0$ corresponds to the traction-free boundary condition and $\mu_{2} / \mu_{1}$ $=\infty$ corresponds to the rigid boundary condition. Figure 5 indicates that the traction-free boundary condition will cause the largest value of stress intensity factor in the transient analysis.

\section{Conclusions}

The phenomena of crack propagation, arrest, and branching are important subjects in the areas of dynamic fracture analysis. The interaction of reflected waves generated from boundaries with the moving crack had only been discussed in experimental works. Experimental results indicated that the reflected waves dominate the stability of crack propagation. It is very important to have the analytical results to investigate this important event. But it seems difficult to obtain the analytical solutions by using the well-known conventional method.

$$
\begin{aligned}
& \operatorname{SIFV}_{m, n}=\operatorname{Re} {\left[\frac{\left[\gamma_{1 / 2}^{*}\left(\eta_{1, m}^{ \pm}\right)\right]^{m} \gamma_{1 / 2}^{*}\left(\eta_{2}^{ \pm}\right) \gamma_{1 / 2}^{*}\left(\eta_{3}^{ \pm}\right) \ldots \gamma_{1 / 2}^{*}\left(\eta_{n+1}^{ \pm}\right)\left( \pm \frac{\partial \eta_{1, m}^{ \pm}}{\partial t_{1}}\right) \ldots\left( \pm \frac{\partial \eta_{n}^{ \pm}}{\partial t_{n}}\right)\left( \pm \frac{\partial \eta_{n+1}^{+}}{\partial t_{n+1}}\right)}{\left(1-\eta_{1, m}^{ \pm} v\right) \alpha_{1+}^{*}\left(\eta_{1, m}^{ \pm}\right)\left(\eta_{1, m}^{ \pm}-\eta_{2}^{ \pm}\right)\left(\eta_{2}^{ \pm}-\eta_{3}^{ \pm}\right) \ldots\left(\eta_{n-1}^{ \pm}-\eta_{n}^{ \pm}\right)\left(\eta_{n}^{ \pm}-\eta_{n+1}^{+}\right)}\right]_{t=\tau} \text { for } n=1,3,5, \ldots } \\
& \operatorname{SIFV}_{m, n}=\operatorname{Im} {\left[\frac{\left[\gamma_{1 / 2}^{*}\left(\eta_{1, m}^{ \pm}\right)\right]^{m} \gamma_{1 / 2}^{*}\left(\eta_{2}^{ \pm}\right) \gamma_{1 / 2}^{*}\left(\eta_{3}^{ \pm}\right) \ldots \gamma_{1 / 2}^{*}\left(\eta_{n+1}^{ \pm}\right)\left( \pm \frac{\partial \eta_{1, m}^{ \pm}}{\partial t_{1}}\right) \ldots\left( \pm \frac{\partial \eta_{n}^{ \pm}}{\partial t_{n}}\right)\left( \pm \frac{\partial \eta_{n+1}^{+}}{\partial t_{n+1}}\right)}{\left(1-\eta_{1, m}^{ \pm} v\right) \alpha_{1+}^{*}\left(\eta_{1, m}^{ \pm}\right)\left(\eta_{1, m}^{ \pm}-\eta_{2}^{ \pm}\right)\left(\eta_{2}^{ \pm}-\eta_{3}^{ \pm}\right) \ldots\left(\eta_{n-1}^{ \pm}-\eta_{n}^{ \pm}\right)\left(\eta_{n}^{ \pm}-\eta_{n+1}^{+}\right)}\right]_{t=\tau} \text { for } n=2,4,6, \ldots } \\
& \eta_{1, m}^{ \pm}=\frac{-h\left(1-b_{1} v\right) t_{1}-(2 m l)^{2} b_{1}^{2} v \pm i 2 m l \sqrt{t_{1}^{2}-b_{1}^{2}\left\{\left[v t_{1}+h\left(1-b_{1} v\right)\right]^{2}+(2 m l)^{2}\right\}}}{h^{2}\left(1-b_{1} v\right)^{2}+(2 m l)^{2}\left(1-b_{1}^{2} v^{2}\right)},
\end{aligned}
$$

$$
\begin{gathered}
\eta_{j}^{ \pm}=\frac{-b_{1}^{2} v l \pm i \sqrt{\left(1-b_{1}^{2} v^{2}\right) t_{j}^{2}-4 l^{2} b_{1}^{2}}}{2 l\left(1-b_{1}^{2} v^{2}\right)}, j=2,3,4, \ldots \\
t_{v, m}=\frac{b_{1}^{2} v h\left(1-b_{1} v\right)+b_{1} \sqrt{h^{2}\left(1-b_{1} v\right)^{2}+(2 m l)^{2}\left(1-b_{1}^{2} v^{2}\right)}}{1-b_{1}^{2} v^{2}}, \\
t_{j}=t-t_{1}-t_{2}-\ldots-t_{j-1}, \quad j=2,3,4, \ldots \\
\gamma_{1 / 2}^{*}(\eta)=\frac{\mu_{1} \alpha_{1}^{*}(\eta)-\mu_{2} \alpha_{2}^{*}(\eta)}{\mu_{1} \alpha_{1}^{*}(\eta)+\mu_{2} \alpha_{2}^{*}(\eta)}
\end{gathered}
$$$$
\alpha_{1}^{*}(\eta)=\alpha_{1+}^{*}(\eta) \alpha_{1-}^{*}(\eta)
$$$$
=\sqrt{b_{1}+\eta\left(1-b_{1} v\right)} \sqrt{b_{1}-\eta\left(1+b_{1} v\right)},
$$$$
\alpha_{2}^{*}(\eta)=\sqrt{b_{2}+\eta\left(1-b_{2} v\right)} \sqrt{b_{2}-\eta\left(1+b_{2} v\right)},
$$$$
t_{r r}=\frac{2 l b_{1}}{\sqrt{1-b_{1}^{2} v^{2}}} \text {. }
$$

We have proposed a powerful superposition methodology and a useful fundamental solution is constructed in this study to solve the problem of a propagating crack in a layered medium. The fundamental solution is the problem of applying an exponentially distributed traction on the propagating crack face and the solution is determined by superposition of the fundamental solution in the Laplace transform domain. The dynamic crack propagation with constant velocity in a configuration of layered medium with a strip is investigated. An explicit and complete result of the dynamic stress intensity factor is obtained in closed form and numerical results are evaluated in detail. The numerical results show that the stress intensity factors induced by reflected waves from the interface are significant.

There still have many unanswered questions in dynamic fracture and this work may provide a useful technique for further investigation in more complicated dynamic fracture problems especially on the crack propagation event. The proposed method in this study has already been extended to solve more difficult in-plane problem of crack propagation with boundary effect, the results will be shown in a future paper.

For the numerical calculation of the transient stress intensity factor, we consider a semi-infinite crack in a layered medium and subjected to a symmetric dynamic concentrated loading on a stationary crack faces at time $t=0$. At nondimensional delay time $t / b_{1} h=1$, the crack starts to propagate with a constant speed $v$ from the semi-infinite crack, the reflected and diffracted waves generated in the layered medium are shown in Fig. 2. The dynamic stress intensity factors of propagating crack for various situations are shown in Figs. 3-5. Figure 3 shows the dynamic stress intensity factor of a single strip for various crack

\section{Acknowledgments}

The authors gratefully acknowledge the financial support of this research by the National Science Council (Republic of China) under Grant NSC 83-0401-E-002-115. Some portions of the manuscript appeared at the 1995 ASME PVP meeting.

\section{References}

Baker, B. R., 1962, "Dynamic Stresses Created by a Moving Crack," ASME Journal of Applied Mechanics, Vol, 29, pp. 449-458. 
Broberg, K. B., 1960, "The Propagation of a Brittle Crack," Arkiv for Fysik, Vol. 18, pp. 159-192.

Craggs, J. W., 1960, "On the Propagation of a Crack in an Elastic-Brittle Solid," Journal of the Mechanics and Physics of Solids, Vol. 8, pp. 66-75.

Freund, L. B., 1972a, "Crack Propagation in an Elastic Solid Subjected to General Loading-I. Constant Rate of Extension," Journal of the Mechanics and Physics of Solids, Vol. 20, pp. 129-140.

Freund, L. B., 1972b, "Crack Propagation in an Elastic Solid Subjected to General Loading-II. Non-uniform Rate of Extension," Journal of the Mechanics and Physics of Solids, Vol. 20, pp. 141-152.

Freund, L. B., 1973, "Crack Propagation in an Elastic Solid Subjected to General Loading--III. Stress Wave Loading," Journal of the Mechanics and Physics of Solids, Vol. 21, pp. 47-61.

Freund, L. B., 1974, "Crack Propagation in an Elastic Solid Subjected to General Loading - IV. Obliquely Incident Stress Pulse," Journal of the Mechanics and Physics of Solids, Vol. 22, pp. 137-146.

Freund, L. B,, 1990, Dynamic Fracture Mechanics, Cambridge University Press, Cambridge, U.K.

Freund, L. B., and Rosakis, A. J., 1992, "The Structure of the Near-tip Field during Transient Elastodynamic Crack Growth," Journal of the Mechanics and Physics of Solids, Vol. 40, pp. 699-719.

Ma, C. C., and Burgers, P., 1986, "Mode III Crack Kinking with Delay Time: An Analytical Approximation," International Journal of Solids and Structures, Vol. 22, pp. 883-899.

Ma, C. C., and Burgers, P., 1987, "Dynamic Mode I and Mode II Crack Kinking Including Delay Time Effects," International Journal of Solids and Structures, Vol. 23, pp. 897-918.

Ma. C. C., and Burgers, P., 1988, "Initiation, Propagation, and Kinking of an Antiplane Crack," ASME Journal of APPLIED MeCHANICS, Vol. 55, pp. 111 119.

Ma, C. C., 1988, "Initiation, Propagation, and Kinking of an In-plane Crack," ASME Journal of APplied Mechanics, Vol. 55, pp. 587-595.
Ma, C. C., 1990, "Dynamic Mixed Mode I-II Crack Kinking under Oblique Stress Wave Loading in Brittle Solids," ASME JouRnAL of APPLIED MECHANICS Vol. 57, pp. 117-127.

Ma, C. C., and Chen, S. K., 1994, "Exact Transient Full Field Analysis of an Antiplane Subsurface Crack Subjected to Dynamic Impact Loading," ASME JOURNAL OF APPLIED MECHANICS, Vol. 61, pp. 649-655.

Ma, C. C., and Ing, Y. S., 1995 "Transient Analysis of Dynamic Crack Propagation with Boundary Effect," ASME Journal of APpLIEd MEchanics, in press. Nilsson, F., 1973, "A Transient Crack Problem for an Infinite Strip under Antiplane Shear," Dynamic Crack Propagation, G. C. Sih, ed., Nordhoff, Dordrecht, The Netherlands, pp. 543-551.

Tsai, C. H., and Ma, C. C., 1992, "Transient Analysis of a Semi-infinite Crack Subjected to Dynamic Concentrated Forces," ASME JoURNAL OF APPLIED ME CHANiCs, Vol. 59, pp. 804-811.

Tsai, C. H., and Ma, C. C., 1993a, "The Transient Analysis of a Subsurface Inclined Crack Subjected to a Buried Dynamic Dilatational Source," International Journal of Solids and Structures, Vol. 30, pp. 399-412.

Tsai, C. H., and Ma, C. C., 1993b, "The Stress Intensity Factor of a Subsurface Inclined Crack Subjected to a Dynamic Impact Loading," International Journal of Solids and Structures, Vol. 30, pp. 2163-2175.

Yang, W., Suo, Z., and Shih, C. F., 1991, "Mechanics of Dynamic Debonding," Proc: $R$. Soc. Lond., Vol. A433, pp. 679-697.

Yang, W., and Shih, C. F., 1994, "Fracture along an Interlayer," International Journal of Solids and Structures, Vol. 31, pp. 985-1002.

Yoffe, E. H., 1951, "The Moving Griffith Crack," Philosophical Magazine, Vol. 42, pp. 739-750.

Yu, H., and Yang, W., 1994, "Mechanics of Transonic Debonding of a Bimaterial Interface: The Anti-plane Shear Case," Journal of the Mechanics and Physics of Solids, Vol. 42, pp. 1789-1802.

Yu, H., and Yang, W., 1995, "Mechanics of Transonic Debonding of a Bimaterial Interface: The In-plane Case," Journal of the Mechanics and Physics of Solids, Vol. 43, pp. 207-232. 